\title{
TÖPLERIAN $\left(L^{2}\right)$-BASES
}

\author{
BY \\ PHILIP HARTMAN AND AUREL WINTNER
}

The following considerations deal with periodic functions $\phi(t)$ having the property that either the sequence $\phi(t), \phi(2 t), \cdots, \phi(n t), \cdots$ or the sequence which results if the constant 1 is adjoined to these functions forms a basis of the $\left(L^{2}\right)$-space over a $t$-interval. By this is meant that $\phi(t)$ itself be of class $\left(L^{2}\right)$ and that, corresponding to every $\epsilon>0$ and to every function $f(t)$ of class $\left(L^{2}\right)$ on the interval, the $\left(L^{2}\right)$-distance from $f(t)$ to

$$
\sum_{n=1}^{K} C_{n} \phi(n t) \quad\left(\text { or } C_{0}+\sum_{n=1}^{K} C_{n} \phi(n t)\right)
$$

be less than $\epsilon$, if $K$ and $K$ constants, $C_{n}$, are suitably chosen.

Since every periodic $\phi(t)$ can be written as the sum of two periodic functions one of which is odd and the other even, there will be no loss of generality in assuming that $\phi(t)$ is either odd or even. In addition, it will be clear from the proofs that the treatment of the case of an even $\phi(t)$ does not differ from that of an odd $\phi(t)$. For this reason, only the latter case will be considered. Then the adjunction of the function 1 becomes unnecessary.

The problem goes back to the formal considerations of Töpler [6](1), a physicist who loc. cit. was concerned with generalized forms of wave analysis.

(Added in proof (23 January 1948). In the paper $A$ class of sequences of functions, Trans. Amer. Math. Soc. vol. 60 (1946) pp. 475-518, some problems dealt with below are considered by D. G. Bourgin along the lines used in [9].)

1. If $\phi_{1}, \phi_{2}, \cdots$ is a sequence of numbers the first of which is not 0 , then a unique sequence ${ }^{*} \phi_{1},{ }^{*} \phi_{2}, \ldots$ is defined by the conditions

$$
{ }^{*} \phi_{1} \phi_{1}=1, \quad \sum_{j k=n}^{*} \phi_{j} \phi_{k}=0, \quad \text { where } \quad n=2,3, \cdots .
$$

In fact, if $n=1$ is included, the first $n$ of the equations (1) represent for ${ }^{*} \phi_{1}, \cdots,{ }^{*} \phi_{n}$ a recursive linear system, the determinant of which is $n$th power of $\phi_{1} \neq 0$. Clearly, (1) is formally equivalent to the generating identity

$$
{ }^{*} \Phi(s) \Phi(s)=1 \text {, }
$$

where

$$
\Phi(s)=\sum_{n=1}^{\infty} \phi_{n} / n^{s}
$$

Presented to the Society, April 26, 1947; received by the editors February 14, 1947.

(1) Numbers in brackets refer to the references cited at the end of the paper. 
and

$$
{ }^{*} \Phi(s)=\sum_{n=1}^{\infty}{ }^{*} \phi_{n} / n^{s}
$$

(these Dirichlet series need not have half-planes of convergence).

Some of the facts concerning the $\left(L^{2}\right)$-bases in question can be collected as follows:

Let $\phi(t)$ be an odd function of period $2 \pi$ and of class $\left(L^{2}\right)$, that is, let

$$
\sum_{m=1}^{\infty}\left|\phi_{m}\right|^{2}<\infty,
$$

where

$$
\phi(t) \sim \sum_{m=1}^{\infty} \phi_{m} \sin m t .
$$

Then, in order that the sequence

$$
\phi(t), \phi(2 t), \cdots, \phi(n t), \cdots
$$

be an $\left(L^{2}\right)$-basis of the $\left(L^{2}\right)$-class on the $t$-interval $(0, \pi)$,

(i) it is necessary and sufficient that the homogeneous equations

$$
\sum_{m=1}^{\infty} \phi_{m} x_{n m}=0 \quad(n=1,2, \cdots)
$$

do not possess within Hilbert's limitation

$$
\sum_{m=1}^{\infty}\left|x_{m}\right|^{2}<\infty
$$

any solution distinct from

$$
x_{1}=x_{2}=\cdots=0 \text {, }
$$

the trivial solution;

(ii) it is necessary that

$$
\phi_{1} \neq 0 \text {, }
$$

whereas

(iii) it is sufficient that $\phi_{1} \neq 0$ and

$$
\sum_{m=1}^{\infty} \sum_{j k=m}\left|{ }^{*} \phi_{j} \phi_{k}\right|^{2}<\infty
$$

(and (12) implies both 


$$
\sum_{n=1}^{\infty}\left|{ }^{*} \phi_{n}\right|^{2}<\infty
$$

and (5) itself); in particular,

(iv) it is sufficient that $\phi_{1} \neq 0$ and, for some $\epsilon>0$,

$$
\phi_{n}=O\left(n^{-1 / 2-c}\right) \text { and }{ }^{*} \phi_{n}=O\left(n^{-1 / 2-c}\right)
$$

as $n \rightarrow \infty$; what is more,

(v) it is sufficient that $\phi_{1} \neq 0$ and

$$
\sum_{m=1}^{\infty}\left|\sum_{j k=m}^{i>N}{ }^{j} \phi_{j} \phi_{k}\right|^{2} \rightarrow 0
$$

as $N \rightarrow \infty$; but

(vi) it is insufficient that just (5) and (11) or, for that matter,

$$
\sum_{m=1}^{\infty}\left|\phi_{n}\right|<\infty
$$

and (11) be satisfied; in fact, the choice $\phi_{1}=1, \phi_{2}=-2, \phi_{3}=\phi_{4}=\cdots=0$ in (6), that is, the trigonometric polynomial

$$
\phi(t)=\sin t-2 \sin 2 t,
$$

supplies a counter-example.

The matrix of the linear system (8) happens to be precisely the $D$-matrix which Toeplitz [7] has associated with the Dirichlet series (3).

Proof of (i). The Fischer-Riesz assignment

$$
x(t) \sim \sum_{m=1}^{\infty} x_{m} \sin m t
$$

establishes a one-to-one correspondence between all functions $x(t)$ of class $\left(L^{2}\right)$ on $(0, \pi)$ and all sequences $x_{1}, x_{2}, \cdots$ satisfying (9). Since (6) implies that

$$
\phi(n t) \sim \sum_{m=1}^{\infty} \phi_{n} \sin m n t,
$$

it is clear from the polarized form of Parseval's relation that the $n$th of the equations (8) is satisfied by the coefficients of (18) if and only if the (nonHermitian) scalar product of $x(t)$ and $\phi(n t)$ vanishes. Consequently, no sequence $x_{1}, x_{2}, \cdots$ satisfying both (9) and each of the equations will be distinct from the trivial sequence $x_{1}=0, x_{2}=0, \cdots$ if and only if the function set (7) has the property that a function $x(t)$ of class $\left(L^{2}\right)$ cannot be orthogonal to each of the functions (7) unless $x(t)=0$ (almost everywhere). Hence, (i) follows from a well known property of Hilbert's space, namely, from the fact 
that a set of functions of class $\left(L^{2}\right)$ forms a basis if and only if no function of class $\left(L^{2}\right)$ which is orthogonal to all functions contained in the set is distinct from the function 0 (almost everywhere).

Proof of (ii). If $\phi_{1}=0$, then the system (8) is readily seen to have the solution

$$
x_{1}=1, \quad x_{2}=x_{3}=\cdots=0 .
$$

Hence, (ii) follows from (i)

Proof of (iii). Clearly, (1) defines one of the sequences $\phi_{1}, \phi_{2}, \cdots$, ${ }^{*} \phi_{1},{ }^{*} \phi_{2}, \cdots$ in terms of the other if and only if $\phi_{1} \neq 0$ and/or ${ }^{*} \phi_{1} \neq 0$. If (12) is assumed, then, since the finite sum representing the $m$ th term of the series (12) is of the form $\left|{ }^{*} \phi_{1} \phi_{m}\right|^{2}+\{\cdots\}$, where $\{\cdots\}$ is a set of non-negative values,

$$
\sum_{m=1}^{\infty}\left|{ }^{*} \phi_{1} \phi_{m}\right|^{2}<\infty
$$

Hence, (5) follows from ${ }^{*} \phi_{1} \neq 0$, which is assumption (11) of (ii) (and (13) is now clear from the fact that both (1) and (12) are symmetric in $\left.\phi,{ }^{*} \phi\right)$.

In view of (i), the assertion of (iii) is equivalent to the statement that (8), (9) and (12) imply (10). In order to verify this implication, replace $n$ in (8) by $n k$, where $k=1,2, \cdots$, and multiply the result by ${ }^{*} \phi_{k}$. Then summation with regard to $k$ gives the infinity of equations

$$
\sum_{k=1}^{\infty}{ }^{*} \phi_{k} \sum_{m=1}^{\infty} \phi_{m} x_{m k n}=0 \quad(n=1,2, \cdots) .
$$

However, since (9), (12) and Schwarz's inequality imply that

$$
\sum_{m=1}^{\infty}\left|x_{m n}\right| \sum_{j k=m}\left|{ }^{*} \phi_{j} \phi_{k}\right|<\infty \quad(n=1,2, \cdots),
$$

these equations can be rearranged into

$$
\sum_{m=1}^{\infty} x_{m n} \sum_{j k=m}^{*} \phi_{j} \phi_{k}=0 \quad(n=1,2, \cdots) .
$$

Hence, (10) follows from (1).

Proof of (iv). If $\epsilon$ in (14) is replaced by $2 \epsilon$, and if (14) is assumed, it is clear that

$$
\sum_{j k=m}\left|{ }^{*} \phi_{j} \phi_{k}\right|=O\left(m^{-1 / 2-2 \epsilon}\right) \sum_{j k=m} 1=O\left(m^{-1 / 2-2 c}\right) d(m),
$$

where $d(m)$ denotes the number of the divisors of $m$. Since, as is well known, $d(m)=O\left(m^{\circ}\right)$ holds for every $\epsilon>0$, this means that the $m$ th term of the series (12) is the square of $O\left(m^{-1 / 2-2 c}\right) O\left(m^{c}\right)=O\left(m^{-1 / 2-c}\right)$. It follows therefore 
from $\sum\left(m^{-1 / 2-\epsilon}\right)^{2}<\infty$ that (iv) is implied by (iii).

Proof of (v). The proof of this refinement of (iii) is similar to that of (iii) itself (and relates to the proof of (iii) itself in about the same way as Mertens' multiplication theorem does to that of Cauchy).

First, if $n$ in (8) is replaced by $j n$, it follows that the equations

$$
\sum_{j=1}^{N}{ }^{*} \phi_{j} \sum_{m=1}^{\infty} \phi_{m} x_{m j n}=0 \quad(n=1,2, \cdots)
$$

hold for arbitrary values $n$ and $N$. Since (6) and (9) imply, by Schwarz's inequality, the absolute convergence of the series occurring in these equations, it is allowed to rearrange the latter into

$$
\sum_{m=1}^{\infty} x_{m n} \sum_{j k=m}^{i \leqslant N}{ }^{N} \phi_{j} \phi_{k}=0 .
$$

On the other hand, from (1),

$$
x_{n}=\sum_{m=1}^{\infty} x_{m n} \sum_{j k=m}^{*} \phi_{j} \phi_{k} .
$$

Hence, by subtraction,

$$
x_{n}=\sum_{m=1}^{\infty} x_{m n} \sum_{j k=m}^{j>N}{ }^{*} \phi_{j} \phi_{k} .
$$

Consequently, if $\epsilon_{N}$ denotes the series (15), then, by Schwarz's inequality,

$$
\left|x_{n}\right|^{2} \leqq \epsilon_{N} \sum_{m=1}^{\infty}\left|\cdot x_{m}\right|^{2}
$$

since $\sum_{m=1}^{\infty}\left|x_{m n}\right|^{2} \leqq \sum_{m=1}^{\infty}\left|x_{m}\right|^{2}$.

If $n$ is fixed and $N \rightarrow \infty$, then, since the assumption of $(v)$ is that $\epsilon_{N} \rightarrow \infty$, the last formula line implies that $x_{n}=0$. Hence, (v) follows from (i).

Proof of (vi). According to (6), the equations belonging to the function (17) are

$$
x_{n}-2 x_{2 n}=0 \quad(n=1,2, \cdots)
$$

and are therefore satisfied by placing $x_{n}=2^{-i}$ or $x_{n}=0$ according as $n$ is the $i$ th power of 2 or is not of the form $2^{i}$, where $i=0,1, \cdots$. Since this sequence $x_{1}, x_{2}, \cdots$ satisfies (9) but is not the trivial solution', (vi) follows from (i).

2. As a further application of (i), the following characterization of the trigonometric sequence

$$
\sin t, \sin 2 t, \cdots, \sin n t, \cdots
$$

will now be deduced: 
(vii) On the interval $(0, \pi)$, the pure harmonic system (20) is the only complete orthogonal system of the form (7), where $\phi(t)$ is odd, of period $2 \pi$ and of class $\left(L^{2}\right)$.

Needless to say, (vii) is meant in the sense that two systems (7), belonging to $\phi(t)=\phi_{\mathrm{I}}(t)$ and $\phi(t)=\phi_{\mathrm{II}}(t)$, are considered as identical if

$$
\phi_{\mathrm{I}}(t) \doteq \text { const. } \phi_{\mathrm{II}}(t) \text { almost everywhere. }
$$

Proof of (vii). If $z^{*}$ denotes the complex conjugate of $z$, the orthogonality of the sequence (7) means that

$$
\int_{0}^{\pi} \phi(j t) \phi^{*}(k t) d t=0 \quad \text { if } j \neq k .
$$

In view of (19) and of the polarized form of Parseval's relation, this is equivalent to

$$
\sum_{m=1}^{\infty} \phi_{k m /(j, k)} \phi_{j m /(j, k)}^{*}=0
$$$$
\text { if } j \neq k \text {, }
$$

where the subscripts are the ratios of the products $\mathrm{km}, \mathrm{jm}$ to the greatest common divisor, $(j, k)$, of $j$ and $k$. In particular, if $j$ is a multiple of $k$, say $j=n k$, then the proviso $j \neq k$ becomes satisfied when $n \neq 1$. Accordingly, since $(j, k)=(n k, k)=k$,

$$
\sum_{m=1}^{\infty} \phi_{m} \phi_{m n}^{*}=0
$$

if $n>1$.

Since an orthogonal system is an $\left(L^{2}\right)$-basis if and only if it is complete, (i) is applicable under the assumptions of (vii). In particular, $\phi_{1} \neq 0$, by (ii). But $\phi_{1} \neq 0$ and the last formula line imply that the system (8) is satisfied by

$$
x_{1}=-\sum_{m=2}^{\infty} \phi_{m} \phi_{m}^{*} / \phi_{1}, \quad x_{2}=\phi_{2}^{*}, \quad x_{3}=\phi_{3}^{*}, \cdots
$$

According to (5), this solution of (8) fulfils (9). Hence, it must be the trivial solution, by (i). In other words, every $x_{n}$ in the last formula line is 0 . Since this means that $\phi_{2}{ }^{*}=\phi_{3}{ }^{*}=\cdots=0$, hence $\phi_{2}=\phi_{3}=\cdots=0$, the expression (6) reduces to

$$
\phi(t) \sim \phi_{1} \sin t .
$$

In view of the completeness of $(20)$ on $(0, \pi)$, this means that $(21)$ holds for

$$
\phi_{\mathrm{I}}(t)=\phi(t), \quad \phi_{\mathrm{II}}(t)=\sin t, \quad \text { and const. }=\phi_{1} .
$$

This proves (vii).

If the system (7) is not an $\left(L^{2}\right)$-basis on $(0, \pi)$, it can be an orthogonal 
system distinct from (20). If it is an orthogonal system on $(0, \pi)$, then the implication

$$
\left\{\sum_{j=1}^{i} C_{j} \phi(j t)=0 \text { p.p. }\right\} \rightarrow\left\{C_{1}=0, \cdots, C_{i}=0\right\}, \text { unless } \phi(t)=0 \text { p.p. }
$$

is trivial for every $i<\infty$, even though the system (7) need not be complete, that is, an $\left(L^{2}\right)$-basis on $(0, \pi)$. On the other hand, $(22)$ is not evident if the system $(7)$ is an $\left(L^{2}\right)$-basis but not orthogonal. But it will now be shown that (22) is then true. As a matter of fact, (22) is true even if (7) fails to be an $\left(L^{2}\right)$-basis. Still more is contained in the following assertion:

(viii) If an odd function $\phi(t)$, of class $\left(L^{2}\right)$ and of period $2 \pi$, is not 0 almost everywhere, then, whether the corresponding sequence $(7)$ is or is not an $\left(L^{2}\right)$-basis on $(0, \pi)$, none of the functions $(7)$ is a limit, in the mean $\left(L^{2}\right)$ on $(0, \pi)$, of linear combinations of all the other functions (7).

This implies, of course, that the functions (7) are linearly independent $\left(L^{2}\right)$ on $(0, \pi)$, which means that $(22)$ is true on $(0, \pi)$.

Proof of (viii). Let $h$ be the index of the first non-vanishing coefficient of (6), that is, let

$$
\phi(t) \sim \sum_{m=h}^{\infty} \phi_{m} \sin m t, \quad \text { where } \phi_{h} \neq 0 .
$$

This $h$ exists, since $\phi(t)$ is not 0 almost everywhere.

Let $i$ be a fixed positive integer, denoting the index of that of the functions (7) for which the impossibility of the $\left(L^{2}\right)$-approximation by linear combinations of the functions $\phi(n t)$, where $n \neq i$, should be proved. If $i=1$, the assertion of (viii) is true. In fact, $\phi(i t)$ then becomes the function (23), and it is clear from (23) that $\sin h t$ is orthogonal to each of the functions $\phi(2 t)$, $\phi(3 t), \cdots$ but is not orthogonal to $\phi(t)=\phi(i t)$. Hence, if $i=1$, the assertion of (viii) follows from standard properties of Hilbert's space. It will therefore be assumed that $i>1$.

With reference to any set of $i-1$ data $C_{1}, \cdots, C_{i-1}$ (a set which, because of $i>1$, is not vacuous), consider the $i-1$ linear equations

$$
\sum_{m<i / n} \phi_{h m} y_{n m}=C_{n}, \quad n=1, \cdots, i-1,
$$

for the $i-1$ unknowns $y_{1}, \cdots, y_{i-1}$. The fixed divisor, $h$, of the subscript of $\phi$ in (24) is the integer defined by (23). The number of the summation values $m$ occurring in the $n$th of the $i-1$ equations $(24)$ is $[i / n]$ or $[i / n]-1$ according as $[i / n]$ is less than or equal to $i / n$, that is, according as $i$ is not or is a multiple of $n$. The matrix of (24) is triangular and all of its diagonal elements are $\phi_{h} \neq 0$. Hence, (24) has a unique solution for arbitrary $C_{n}$.

Choose $C_{n}$ to be $-\phi_{i n / n}$ or 0 according as the quotient $i h / n$ is or is not 
an integer, and denote by $y_{1}, \cdots, y_{i-1}$ the values which are then defined by (24); so that

$$
\sum_{m<i / n} \phi_{h m} y_{n m}+\phi_{h i / n}=0, \quad n=1, \cdots, i-1,
$$

if the last $\phi$ denotes 0 when its subscript is not an integer. In terms of this set of $i-1$ values $y_{j}$, define an infinite sequence $x_{1}, x_{2}, \cdots$ as follows: $x_{k}$ is 0 unless $k$ is a multiple of $h$, whereas in the remaining case the complex conjugate of $x_{k}$ is $y_{k / h}, 1$ or 0 according as $k$ is less than, equal to or greater than the integer $i h$. Then (18) defines a function $x(t)$ of class $\left(L^{2}\right)$; in fact, (18) becomes a trigonometric polynomial. If it is verified that the (Hermitian) scalar product of this trigonometric polynomial and of the $n$th of the functions (7) is 0 or distinct from 0 according as $n \neq i$ or $n=i$, then the assertion of (viii) for the $i$ th of the functions (7) follows for the same reason as it did in the case $i=1$ (the latter has been considered before (24) and is excluded now).

If the polarized form of Parseval's relations is applied to (19) and to the complex conjugate of the trigonometric polynomial (18) in the same way as at the beginning of the proof of (vii), it is seen from the definition of $x_{1}, x_{2}, \ldots$ in terms of the $i-1$ values $y_{j}$ assigned by the last formula line that the scalar product of $x(t)$ and $\phi(n t)$ is a sum of 0 's (hence 0 ) or the expression on the left of the last formula line (hence 0 ) according as $n>i$ or $n<i$, and that it has the value $\phi_{h}$ if $n=i$. But $\phi_{h} \neq 0$, by (23). Hence, $x(t)$ and $\phi(n t)$ are orthogonal or not according as $n \neq i$ or $n=i$. This proves (viii).

3. In (i)-(vi), the sequence (7) is meant to be an $\left(L^{2}\right)$-basis on $(0, \pi)$ if, corresponding to every $x(t)$ of class $\left(L^{2}\right)$ on $(0, \pi)$, it is possible to find a $K=K(\epsilon)$ and $K(\epsilon)$ constants $C_{n}(\epsilon)$ satisfying

$$
\int_{0}^{\pi}\left|x(t)-\sum_{n=1}^{K(\epsilon)} C_{n}(\epsilon) \phi(n t)\right|^{2} d t<\epsilon
$$

when $\epsilon>0$ is given arbitrarily. In contrast to this definition of a mere $\left(L^{2}\right)$ basis $(7)$ on $(0, \pi)$, let $(7)$ be called a Fourier $\left(L^{2}\right)$-basis on $(0, \pi)$ if, corresponding to every $x(t)$ of class $\left(L^{2}\right)$ on $(0, \pi)$, the $K(\epsilon)$ constants $C_{n}(\epsilon)$ occurring in (25) can be chosen independent of $\epsilon$. In other words, (7) will be called a Fourier $\left(L^{2}\right)$-basis on $(0, \pi)$ if there belongs to every $x(t)$ of class $\left(L^{2}\right)$ on $(0, \pi)$ a sequence of constants $c_{n}$ satisfying

$$
\int_{0}^{x}\left|x(t)-\sum_{n=1}^{k} c_{n} \phi(n t)\right|^{2} d t \rightarrow 0 \quad \text { as } k \rightarrow \infty,
$$

where every $c_{n}$ is independent of $k$.

The difference between the notion of an $\left(L^{2}\right)$-basis $(7)$ and the more restricted notion of a Fourier $\left(L^{2}\right)$-basis (7) (which, in view of (vii), are beforehand identical only in the classical case) has been emphasized in $[10$, p. 764]. 
Due to the criteria (i)-(vi) for mere $\left(L^{2}\right)$-bases, it will now be possible to study this anomaly in some detail, if use is made of the criterion proved in [10] for Fourier $\left(L^{2}\right)$-basis. The latter criterion becomes equivalent to (xiv) below by virtue of the following fact:

(ix) If $\phi(t)$ is an odd function, of class $\left(L^{2}\right)$ and of period $2 \pi$, satisfying $\phi_{1} \neq 0$ in (6), and if $x(t)$ is a fixed function, of class $\left(L^{2}\right)$ on $(0, \pi)$, corresponding to which there exists at least one sequence $c_{1}, c_{2}, \cdots$ satisfying (26), then there exists just one such sequence for the given $x(t)$; in fact, the values of $c_{1}, c_{2}, \cdots$ are then determined by

$$
x_{n}=\sum_{d \mid n} c_{d} \phi_{n / d} \quad\left(\phi_{1} \neq 0\right)
$$

or, equivalently, by

$$
c_{n}=\sum_{d \mid n} x_{d} * \phi_{n / d}
$$

$\left({ }^{*} \phi_{1} \neq 0\right)$

where $x_{n}, \phi_{n},{ }^{*} \phi_{n}$ are defined by (18), (6), (1) respectively.

Proof of (ix). This is the same as in [10]. In fact, (18) means that

$$
\pi x_{n} / 2=\int_{0}^{\pi} x(t) \sin n t d t .
$$

Hence, (27) follows from (26) and (19) by strong convergence. This proves (ix), since (28) is equivalent to (27) by virtue of (1).

For certain, rather than for all, functions $x(t)$ of class $\left(L^{2}\right)$, the question as to the existence of a sequence $c_{1}, c_{2}, \ldots$ satisfying (26) has an arithmetical origin and goes back to Riemann. In this connection, cf. [4], where other references are given.

Besides (ix), the following fact which proves to be a mere restatement of the corollary, (22), of (viii), will also be needed:

(x) If $\phi(t)$ is an odd function, of period $2 \pi$ and of class $\left(L^{2}\right)$, and if $x(t)$ is any function of class $\left(L^{2}\right)$ on $(0, \pi)$, then, unless $\phi(t)=0$ (almost everywhere), there belongs to every $k$ just one set of $k$ constants $c_{1}=c_{1}(k), \cdots, c_{k}=c_{k}(k)$ minimizing the integral

$$
\int_{0}^{\pi}\left|x(t)-\sum_{n=1}^{k} c_{n} \phi(n t)\right|^{2} d t .
$$

Proof of (x). The integral (29) is a quadratic polynomial in the $k$ variables $c_{1}, \cdots, c_{k}$ and their complex conjugates. Hence, $(\mathrm{x})$ will be proved if it is shown that the quadratic part of this polynomial, namely, the non-negative definite Hermitian form

$$
\int_{0}^{x}\left|\sum_{n=1}^{k} c_{n} \phi(n t)\right|^{2} d t
$$


is positive definite unless $\phi(t)$ vanishes almost everywhere. But the discriminant of this Hermitian form is the Gram determinant

$$
\operatorname{det}\left(\int_{0}^{\pi} \phi_{m}(t) \phi_{n}^{*}(t) d t\right) \quad(m, n=1, \cdots, k),
$$

where $\phi_{n}^{*}(t)$ denotes the complex conjugate of

$$
\phi_{n}(t)=\phi(n t) .
$$

On the other hand, $k$ functions $\phi_{n}(t)$ of class $\left(L^{2}\right)$ on $(0, \pi)$ are known to be linearly independent (almost everywhere) if and only if the determinant (30) vanishes. Hence, $(x)$ follows from (22),

4. Since (7) cannot be a Fourier $\left(L^{2}\right)$-basis without being an $\left(L^{2}\right)$-basis, (ii) implies that $\phi_{1} \neq 0$ is necessary for a Fourier $\left(L^{2}\right)$-basis. Criteria for a Fourier $\left(L^{2}\right)$-basis, which correspond to the criteria (i), (iii), (iv), (v) for a mere $\left(L^{2}\right)$-basis, can be formulated as follows:

Let $\phi(t)$ be an odd function of period $2 \pi$ and of class $\left(L^{2}\right)$, that is, let (6) define a sequence $\phi_{1}, \phi_{2}, \cdots$ satisfying (5). Then, in order that the functions (7) form a Fourier $\left(L^{2}\right)$-basis on $(0, \pi)$,

(xi) it is necessary and sufficient that $\phi_{1} \neq 0$ and that the sequence ${ }^{*} \phi_{1}$, ${ }^{*} \phi_{2}, \cdots$, which is then defined by (1), be such that, if $x=\left(x_{1}, x_{2}, \cdots\right)$ is any fixed point of Hilbert's space,

$$
\Phi_{k}(x) \rightarrow 0 \text { as } k \rightarrow \infty,
$$

where $\Phi_{k}(x)$ denotes, for $k=1,2, \cdots$, the Hermitian form

$$
\Phi_{k}(x)=\sum_{m=1}^{\infty}\left|\sum_{n=1}^{\infty} x_{n} \sum_{n d \mid m}^{n d>k}{ }^{2} \phi_{d} \phi_{m /(n d)}\right|^{2}
$$

(with the understanding that the convergence of each of the series (32) at every point of Hilbert's space or, equivalently, the boundedness of each of bilinear forms

$$
\sum_{n=1}^{\infty} \sum_{m=1}^{\infty} x_{n} y_{m} \sum_{n d \mid m}^{n d>k} *_{\phi_{d}} \phi_{m /(n d)}
$$

where $k=1,2, \cdots$, is part of the criterion);

(xii) it is necessary that

$$
{ }^{*} \phi_{n} \rightarrow 0 \text { as } n \rightarrow \infty ;
$$

what is more,

(xiii) it is necessary that

$$
\sum_{n=1}^{\infty}\left|\sum_{d \mid n}^{d>k}{ }^{*} \phi_{d} \phi_{n / d}\right|^{2} \rightarrow 0 \text { as } k \rightarrow \infty
$$


(and that the series (35) be convergent for every $k$ );

(xiv) it is sufficient that $\phi_{1}=0$ and that the Dirichlet series (3), (4) be convergent in the half-plane $\sigma>0$ and be such as to represent there functions $\Phi(s)$, ${ }^{*} \Phi(s)$ satisfying

$$
\underset{\sigma>0}{\operatorname{l.u.b}}|\Phi(s)|<\infty
$$

and

$$
\left.\underset{\sigma>0}{\text { l.u.b. }}\right|^{*} \Phi(s) \mid<\infty \text {, that is, } \underset{\sigma>0}{\text { g.l.b. }}|\Phi(s)|>0, c f .(2) \text {. }
$$

Proof of (xi). It is clear from (ix) and (x) that, if $x(t)$ is a fixed function of class $\left(L^{2}\right)$ on $(0, \pi)$, that is, if $x_{1}, x_{2}, \cdots$ in (18) is a fixed sequence satisfying (9), then (26) holds for some sequence $c_{1}, c_{2}, \ldots$ if and only if zero is the limit, as $k \rightarrow \infty$, of the quadratic expression which results if $c_{n}$ is substituted from (28) into (9). But the expression which thus results for a fixed $k$ is seen to be the Hermitian form (32). Hence, (xi) follows from the fact that the definition of a Fourier $\left(L^{2}\right)$-basis involves every point $x=\left(x_{1}, x_{2}, \cdots\right)$ of Hilbert's space.

Proof of (xii). The inequality

$$
\left|{ }^{*} \phi_{k+1} \phi_{1}\right|^{2} \leqq \sum_{n=1}\left|\sum_{d \mid n}^{d>k}{ }^{*} \phi_{d} \phi_{n / d}\right|^{2}
$$

is obvious for arbitrary values ${ }^{*} \phi_{n}, \phi_{n}$ and for every $k$. Hence, if (xiii) is granted for a moment, it follows from (35) that

$$
\left|{ }^{*} \phi_{k+1} \phi_{1}\right|^{2} \rightarrow 0 \text { as } k \rightarrow \infty \text {. }
$$

In view of $\phi_{1} \neq 0$, this proves (34).

Proof of (xiii). According to (xi), condition (31) is necessary and sufficient, if $x_{1}, x_{2}, \cdots$ in (31) is an unspecified sequence satisfying (9). Hence, there results a necessary condition if (38) is required for the particular sequence

$$
x_{1}=1, \quad x_{2}=x_{3}=\cdots=0 .
$$

But (32) shows that (31) then becomes precisely (35), the assertion of (xiii).

The proof of the remaining criterion, (xiv), will be omitted, since (xiv) is contained in the following theorem, proved in [10]:

(xv) Let $\phi_{1}, \phi_{2}, \cdots$, where $\phi_{1} \neq 0$, be a sequence of numbers for which both Dirichlet series $\Phi(s),{ }^{*} \Phi(s)$, defined by (3), (4) and (1), are convergent in the half-plane $\sigma>0$ and satisfy both (36) and (37). Then (6) defines a function of class $\left(L^{2}\right)$ corresponding to which the sequence (7) is a Fourier $\left(L^{2}\right)$-basis on $(0, \pi)$.

In fact, if $x(t)$ is any function of class $\left(L^{2}\right)$ on $(0, \pi)$, the sequence $c_{1}, c_{2}, \cdots$ 
satisfying (26) is uniquely determined by (28), where the data $x_{1}, x_{2}, \cdots$ are given by (18).

In addition, if $x_{1}, x_{2}, \cdots$ is any sequence possible in (18), that is, any sequence satisfying (5), then

$$
\sum_{m=1}\left|c_{m}\right|^{2}<\infty
$$

holds by virtue of (29). Conversely, if $c_{1}, c_{2}, \ldots$ is any sequence satisfying (38), then (9) holds by virtue of (28) and belongs, via (18), to a function $x(t)$, of class $\left(L^{2}\right)$, satisfying (26).

It remains undecided whether the sufficient criterion supplied by (xiv) for a Fourier $\left(L^{2}\right)$-basis is necessary as well. The purpose of the following considerations is to fill into this gap so much that the notions of an $\left(L^{2}\right)$ basis and of a Fourier $\left(L^{2}\right)$-basis become provably distinct.

5. First, the proof of (xv) indicates that the assumptions of (xiv) can hardly be relaxed. In this regard, the following negation should be mentioned:

(xvi) Corresponding to every $\epsilon>0$, there exists a sequence of numbers $\phi_{1} \neq 0, \phi_{2}, \phi_{3}, \cdots$ having the following properties: The Dirichlet series (3), (4) are convergent in the respective half-plane $\sigma>0, \sigma>\epsilon$ and satisfy (36) and

$$
\underset{\sigma>e}{\text { l.u.b. }}\left|{ }^{*} \Phi(s)\right|<\infty \text {, that is, } \underset{\sigma>e}{\operatorname{g.l.b.}}|\Phi(s)|>0 \text {, }
$$

cf. (2), but (6) defines a periodic continuous function for which the sequence (7) fails to be an $\left(L^{2}\right)$-basis on $(0, \pi)$.

In other words, the difference between $\epsilon=0$ and any $\epsilon>0$ is so great that, while the former case necessitates, by (xiv), a Fourier $\left(L^{2}\right)$-basis, the latter is compatible with no $\left(L^{2}\right)$-basis.

Proof of (xvi). Define (6) by

$$
\phi(t)=\sin t-q \sin 2 t,
$$

where $q$ is a constant. Thus (17) results if $q=2$. Correspondingly, the equations (8) belonging to an arbitrary $q$ result if the 2 is replaced by $q$ in the equations given in the proof of (vi). Thus it is clear that, for the same reasons as in the case $q=2$ of (vi), the sequence (7) fails to be an $\left(L^{2}\right)$-basis on $(0, \pi)$ if $q>1$. On the other hand, from (3), (6) and the last formula line,

$$
\Phi(s)=1-q / 2^{s} ;
$$

so that (3) is convergent everywhere and satisfies (27). Finally, from (2),

$$
{ }^{*} \Phi(s)=\left(1-q / 2^{s}\right)^{-1}=\sum_{k=0} q^{k} / 2^{k s} .
$$

This is an ordinary Dirichlet series which is absolutely convergent in the halfplane $\sigma>\log _{2} q$. Hence, (xvi) follows by letting $q \rightarrow 1+0$.

In order to illustrate the limitations imposed by (xvi) on $\phi(t)$, let (6) be 
the generalized Bernoullian function

$$
\phi_{\lambda}(t)=\sum_{m=1}^{\infty} m^{-\lambda} \sin m t,
$$

where $\lambda$ need not be a real (and still less an odd) integer. This is a convergent Fourier series $(L)$ if $\Re \lambda>0$. Clearly, (5) is satisfied if and only if $\Re \lambda>1 / 2$, and this trivial necessary condition turns out to be sufficient in order that (7) be a mere $\left(L_{2}\right)$-basis on $(0, \pi)$. This fact, proved in the last of the papers listed in [9, pp. 572-573], can be concluded from (iii) also. On the other hand, in order to conclude from (xvi) that (7) is a Fourier $\left(L^{2}\right)$-basis on $(0, \pi)$, it is necessary (and sufficient) to replace $\Re \lambda>1 / 2$ by $\Re \lambda>1$. In fact, (6) and the last formula line show that the Dirichlet series (3) now becomes

$$
\Phi(s)=\sum_{m=1}^{\infty} m^{-\lambda} / m^{s}=\zeta(s+\lambda) ;
$$

so that, from (2),

$$
{ }^{*} \Phi(s)=1 / \zeta(s+\lambda)=\sum_{m=1}^{\infty} \mu(m) / m^{s+\lambda},
$$

where $\mu(m)$ is Möbius' factor. Hence the assumptions of (xvi) are satisfied or violated by both (3) and (4) according as $\Re \lambda>1$ is or is not assumed.

For arithmetical reasons, the necessity of the latter limitation is a more involved question than what is needed in the proof of

(xvii) There exist odd functions $\phi(t)$, of period $2 \pi$ and of class $\left(L^{2}\right)$, for which the sequence $(7)$ is an $\left(L^{2}\right)$-basis, but fails to be a Fourier $\left(L^{2}\right)$-basis, on $(0, \pi)$; in fact, the trigonometric polynomial

$$
\phi(t)=\sin t-\sin 2 t
$$

is such $a \phi(t)$.

Proof of (xvii). According to (6) and (40),

$$
\phi_{1}=1, \quad \phi_{2}=-1, \quad \phi_{3}=\phi_{4}=\cdots=0 .
$$

Hence, the equations (8) reduce to $x_{n}-x_{2 n}=0(n=1,2, \cdots)$. Accordingly, $x_{n}=x_{2 n}=x_{4 n}=x_{8 n}=\cdots$. Consequently, (10) is implied by (9). It follows therefore from (i) that ( 7$)$ is an $\left(L^{2}\right)$-basis on $(0, \pi)$.

On the other hand, since the Dirichlet series (3) reduces to

$$
\Phi(s)=1 / 1^{s}-1 / 2^{s}+0+0+\cdots=1-2^{-s},
$$

(2) and (4) show that

$$
\sum_{n=1}^{\infty} *_{\phi_{n}} / n^{s}=\left(1-2^{-s}\right)^{-1}=\sum_{k=0}^{\infty} 1 / 2^{k s} .
$$


Since this implies that (34) is violated, it follows from (xii) that ( 7 ) cannot be a Fourier $\left(L^{2}\right)$-basis on $(0, \pi)$.

6. The sufficient criterion of (xiv) for a Fourier $\left(L^{2}\right)$-basis, when compared with the failure claimed by (xvi) for a mere $\left(L^{2}\right)$-basis, raises the question whether or not there exist sufficient criteria for an $\left(L^{2}\right)$-basis which involve, as (xiv) does, Dirichlet series only. It turns out that such criteria result if only one of the two conditions, (36) and (37), of (xiv) is required, except that, in the case of (36), a supplementary restriction is necessitated by (vi). This pair of sufficient criteria for $\left(L^{2}\right)$-bases is the content of (xxi) and (xix) below.

The proof will require the following refinement of $(\mathrm{v})$ :

(xviii) The assertion of (v) remains true if its assumption (15), that is, the assumption

$$
\sum_{n=1}^{\infty}\left|\sum_{d \mid n}^{d>k} *_{d} \phi_{n / d}\right|^{2}=o(1)
$$

where $k \rightarrow \infty$, is relaxed to

$$
\sum_{n=1}^{\infty}\left|\sum_{d \mid n}^{d>k} *_{d} \phi_{n / d}\right|^{2}=O(1)
$$

that is, to

$$
\sum_{n=1}^{\infty}\left|\sum_{d \mid n}^{d \leq k}{ }^{k} \phi_{d} \phi_{n / d}\right|^{2}=O(1)
$$

Proof of (xviii). Let $x_{1}, x_{2}, \cdots$, be any sequence satisfying (9) and (8). Then, if $k$ and $n$ are arbitrary,

$$
\sum_{m=1}^{k} * \phi_{m} \sum_{j=1} \phi_{j} x_{m n j}=0 .
$$

Clearly, this can be written in the form

$$
\sum_{j=1}^{\infty}\left(\sum_{m \mid j}^{m \leqslant k}{ }^{k} \phi_{m} \phi_{j / m}\right) x_{j n}=0 .
$$

In view of (1), this in turn is equivalent to

$$
x_{n}+\sum_{j=k+1}^{\infty}\left(\sum_{m \mid j}^{m \leq k}{ }^{*} \phi_{m} \phi_{j / m}\right) x_{j n}=0 .
$$

Hence, by Schwarz's inequality,

where

$$
\left|x_{n}\right|^{2} \leqq C_{k}(n) \sum_{j=k+1}^{\infty}\left|x_{j n}\right|^{2} \leqq C_{k}(n) \sum_{m=k+1}^{\infty}\left|x_{m}\right|^{2},
$$




$$
C_{k}(n)=\sum_{j=k+1}^{\infty}\left|\sum_{m \mid j}^{m \leqq k} * \phi_{m} \phi_{j / m}\right|^{2} \leqq \sum_{j=1}^{\infty}\left|\sum_{m \mid j}^{m \leqq k} * \phi_{m} \phi_{j / m}\right|^{2} .
$$

But the last sum is identical with the sum on the left of (42). Hence, the last two formula lines and (42) imply that

$$
\left|x_{n}\right|^{2} \leqq \text { const. } \sum_{m=k+1}^{\infty}\left|x_{m}\right|^{2}
$$

If $k \rightarrow \infty$ when $n$ is fixed, it follows that $x_{n}=0$, where $n=1,2, \cdots$. In view of (i), this completes the proof of (xviii).

The two criteria announced before (xviii) are the third and the first of the following four assertions:

Let $\phi_{1}, \phi_{2}, \cdots$ be a sequence of numbers satisfying

$$
\phi_{1} \neq 0 \text { and } \sum_{m=1}^{\infty}\left|\phi_{m}\right|^{2}<\infty,
$$

and let ${ }^{*} \phi_{n}, \Phi(s),{ }^{*} \Phi(s), \phi(t)$ be defined by (1), (3), (4), (6) respectively. Then, in order that the functions (7) form an $\left(L^{2}\right)$-basis on $(0, \pi)$,

(xix) it is sufficient that

$$
\sum_{m=1}^{\infty}\left|{ }^{*} \phi_{m}\right|^{2}<\infty
$$

and that the Dirichlet series

$$
\Phi(s) \text { converges for } \sigma>0, \text { and } \underset{\sigma>0}{\operatorname{l.u} . \mathrm{b} .}|\Phi(s)|<\infty \text {; }
$$

(xx) it is insufficient that (44), without (43), be satisfied:

(xxi) it is sufficient that the Dirichlet series

$$
{ }^{*} \Phi(s) \text { converges for } \sigma>0 \text {, and } \underset{\sigma>0}{\operatorname{l.u} . \mathrm{b} .}\left|{ }^{*} \Phi(s)\right|<\infty \text {; }
$$

(xxii) it is necessary that both Dirichlet series $\Phi(s),{ }^{*} \Phi(s)$ be absolutely convergent for $\sigma>1 / 2$ and that

$$
\Phi(s) \neq 0 \text { and } * \Phi(s) \neq 0 \text { when } \sigma>1 / 2 \text {. }
$$

The dissymmetry of (xix) and (xxi), emphasized by (xx), is apparent only, since what corresponds to the restriction (43) of (xix) in (xxi) is the restriction (7), assumed from the beginning. The symmetry in the duals (xix), (xxi) is carried even further by the following fact:

(xxiii) The assumptions of (xxi) imply that (43) is satisfied and that

$$
{ }^{*} \phi(t),{ }^{*} \phi(2 t), \cdots,{ }^{*} \phi(n t), \cdots,
$$

defined by (43) and 


$$
{ }^{*} \phi(t) \sim \sum_{m=1}{ }^{*} \phi_{m} \sin m t,
$$

form an $\left(L^{2}\right)$-basis on $(0, \pi)$.

However, the symmetry is only in the wording, rather than in the proof, of (xix) and (xxi), since (xxi) turns out to be deeper than (xix).

Proof of (xix). According to Toeplitz [7], the assumption (44) is equivalent to the boundedness of the infinite bilinear " $D$-form" which he associates with the Dirichlet series $\Phi(s)$. Since a bilinear form having the matrix $A$ is bounded if and only if the Hermitian form belonging to $A A^{*}$ (and/or $A^{*} A$ ) is, it follows from Toeplitz's definition (loc. cit.) of the $D$-form of $\Phi(s)$ that (44) is equivalent to the existence of a constant satisfying

$$
\sum_{n=1}^{\infty}\left|\sum_{d \mid n} \phi_{n / d} x_{d}\right|^{2} \leqq \text { const. } \sum_{m=1}^{\infty}\left|x_{m}\right|^{2},
$$

where $\left(x_{1}, x_{2}, \cdots\right)$ is any point of Hilbert's space.

For a fixed $k$, choose $x_{1}={ }^{*} \phi_{1}, \cdots, x_{k}={ }^{*} \phi_{k}, x_{k+1}=x_{k+2}=\cdots=0$. Then, from (47),

$$
\sum_{n=1}^{\infty}\left|\sum_{d \mid n}^{d \leq k}{ }^{k} \phi_{d} \phi_{n / d}\right|^{2} \leqq \text { const. } \sum_{m=1}^{k}\left|{ }^{*} \phi_{m}\right|^{2} .
$$

It follows therefore from the assumption (43) (which has not been used thus far) that (42) is satisfied. In view of (xviii), this completes the proof of (xix).

Proof of (xx). According to (6) and (3), the Dirichlet series $\Phi(s)$ belonging to (17) is $\Phi(s)=1-2 / 2 s+0+0+\ldots$. Hence, $(x x)$ follows from (vi).

Proof of (xxi). According to Bohr [1], the abscissa of uniform convergence of an ordinary Dirichlet series is identical with abscissa of bounded regularanalyticity of the function represented by the Dirichlet series (provided that the latter does not diverge everywhere). Hence, (45) implies that the function ${ }^{*} \Phi(s)={ }^{*} \Phi(\sigma+i t)$ of $t$ is uniformly almost-periodic on every line $\sigma>0$, and that the Fourier expansion of this almost-periodic function of $t$ is supplied precisely by the Dirichlet series. Consequently, the Cesàro sums of this Fourier expansion are uniformly bounded for $-\infty<t<\infty$ when $\sigma>0$ is fixed and, in view of the second of the assumptions (45), this uniform boundedness is uniform in $\sigma(>0)$ as well; so that

$$
\left|{ }^{*} \Phi^{k}(s)\right|<\text { const., }
$$

where $k=1,2, \cdots$ and $\sigma>0$,

if ${ }^{*} \Phi^{k}(s)$ denotes the Cesàro sum

$$
\begin{aligned}
{ }^{*} \Phi^{k}(s)=\sum_{n_{1}=0}^{k} \cdots \sum_{n_{k}=0}^{k}\left(1-n_{1} / k\right) \cdots\left(1-n_{k} / k\right)^{*} \phi_{j} / j^{s}, & \\
j & =p_{1}^{n_{1}} \cdots p_{k}^{n_{k}},
\end{aligned}
$$


where the $p$ 's denote primes and the $n$ 's their multiplicities in $j$ (cf. [2, pp. 73-75]). Let (49), a terminating Dirichlet series for every $k$, be denoted by

$$
{ }^{*} \Phi^{k}(s)=\sum_{m=1}^{\infty}{ }^{k} \phi_{m} / m^{s}
$$

(so that ${ }^{k} \phi_{m}=0$ if $m$ is large enough with reference to $k$ ).

According to (48), the Dirichlet series (50), where $k=1,2, \cdots$, are (convergent and) uniformly bounded in the half-plane $\sigma>0$. In view of Toeplitz's criterion for the bound of a $D$-form, this means (cf. [7]) that the sequence of the infinite bilinear $D$-forms belonging to the sequence of the Dirichlet series (50) is uniformly bounded (with respect to $k$ ); so that, corresponding to (47),

$$
\sum_{n=1}^{\infty}\left|\sum_{d \mid n}{ }^{k} \phi_{n / d} x_{d}\right|^{2} \leqq \text { const. } \sum_{m=1}^{\infty}\left|x_{m}\right|^{2},
$$

where the constant is independent of $k$ and of the choice of the point $\left(x_{1}, x_{2}, \cdots\right)$ in Hilbert's space (cf. [5, p. 304]).

The last inequality, when applied to $x_{n}=\phi_{n}$, gives

$$
\sum_{n=1}^{\infty}\left|\sum_{d \mid n}{ }^{k} \phi_{n / d} \phi_{d}\right|^{2} \leqq \text { const. } \sum_{m=1}^{\infty}\left|\phi_{m}\right|^{2}=\text { const. }<\infty,
$$

by (5). Due to (51), the proof of (xxi) can now be completed along the lines of the proof of (xviii), as follows:

If $x_{1}, x_{2}, \ldots$ is a sequence satisfying (9) and (8), and if $k$ and $n$ are arbitrary, then

$$
\sum_{n=1}^{\infty}{ }^{k} \phi_{m} \sum_{j=1}^{\infty} \phi_{j} x_{j m n}=0
$$

Since all but a finite number of the coefficients ${ }^{k} \phi_{1},{ }^{k} \phi_{2}, \cdots$ vanish if $k$ is fixed, this identity can be rearranged into

$$
\sum_{m=1}^{\infty}\left(\sum_{d \mid m}{ }^{k} \phi_{m / d} \phi_{d}\right) x_{m n}=0
$$

Hence, if $h$ is arbitrary,

$$
\sum_{m=1}^{h}\left(\sum_{d \mid m}{ }^{k} \phi_{m / d} \phi_{d}\right) x_{m n}=-\sum_{m=h+1}^{\infty}\left(\sum_{d \mid m}{ }^{k} \phi_{m / d} \phi_{d}\right) x_{m n} .
$$

Consequently, by Schwarz's inequality,

$$
\left|\sum_{m=1}^{h}\left(\sum_{d \mid m}{ }^{k} \phi_{m / d} \phi_{d}\right) x_{m n}\right|^{2} \leqq C^{k}(h) \sum_{m=h+1}^{\infty}\left|x_{m n}\right|^{2},
$$


where

$$
C^{k}(h)=\sum_{m=h+1}^{\infty}\left|\sum_{d \mid m}{ }^{k} \phi_{m / d} \phi_{d}\right|^{2} \leqq \sum_{m=1}^{\infty}\left|\sum_{d \mid m}{ }^{k} \phi_{m / d} \phi_{d}\right|^{2} .
$$

Since the last series is the one occurring on the left of (51), the last two formula lines imply that

$$
\left|\sum_{m=1}^{h}\left(\sum_{d \mid m}{ }^{k} \phi_{m / d} \phi_{d}\right) x_{m n}\right|^{2} \leqq \text { const. } \sum_{m=h+1}^{\infty}\left|x_{m}\right|^{2}
$$

where the constant is independent of the three variables $h, k, n$.

On the other hand, a comparison of (50) with (49) shows that

$$
{ }^{k} \phi_{m} \rightarrow{ }^{*} \phi_{m} \text { as } k \rightarrow \infty \text {, }
$$

if $m$ is fixed. Hence, if $h$ and $n$ are kept fixed in the last inequality, it follows, by letting $k \rightarrow \infty$, that

$$
\left|\sum_{m=1}^{h}\left(\sum_{d \mid m}{ }^{*} \phi_{m / d} \phi_{d}\right) x_{m n}\right|^{2} \leqq \text { const. } \sum_{m=h+1}^{\infty}\left|x_{m}\right|^{2} .
$$

According to (1), this can be reduced to

$$
\left|x_{n}\right|^{2} \leqq \text { const. } \sum_{m=h+1}^{\infty}\left|x_{m}\right|^{2}
$$

It follows therefore from (9), by letting $h \rightarrow \infty$ while $n$ is fixed, that $x_{n}=0$, that is, that (10) is a necessity. In view of (i), this completes the proof of (xxi).

Proof of (xxii). In view of Schwarz's inequality, it is clear from (5) and (3) that the Dirichlet series $\Phi(s)$ is absolutely convergent in the half-plane $\sigma>1 / 2$. Since the constant term, $\phi_{1}$, of $\Phi(s)$ is supposed to be distinct from 0 , it follows from (2) and from well known theorems on reciprocation, that the ordinary Dirichlet series of ${ }^{*} \Phi(s)$ must converge absolutely in the half-plane $\sigma>1 / 2$ if the function $\Phi(s)$ does not vanish there, that is, if the first of the two assertions (46) is true. On the other hand, the second of the assertions (46) is obvious from (2). Hence, (xxii) will be proved if it is shown that $\Phi(s)$ cannot vanish in the half-plane $\sigma>1 / 2$. But this can be concluded, by an argument applied in [8] in a finer context, as follows:

Suppose that $\Phi(s)$ has a zero, $s$, in the half-plane $\sigma>1 / 2$. Then, if $x_{n}=n^{-s}$, condition (9) is satisfied and, although (10) does not hold, the equations (8) are fulfilled. In fact, by the definition of $x_{n}$ and of $\Phi(s)$,

$$
\sum_{m=1}^{\infty} \phi_{m} x_{n m}=\sum_{m=1}^{\infty} \phi_{m} /(n m)^{s}=\Phi(s) / n^{s}=0
$$


since $\Phi(s)=0$. It follows therefore from (i) that (7) cannot be an $\left(L^{2}\right)$-basis on $(0, \pi)$. This proves (xxii).

Proof of (xxiii). It is sufficient to show that the assumptions of (xxi) imply (43). For, if this is shown, (xxiii) follows (xxi) for reasons of symmetry, the dual of (43) being (5). Accordingly, it is sufficient to ascertain that (45) implies (43).

As seen above, (44) implies (47). Hence, for reasons of symmetry, (45) implies

$$
\sum_{n=1}\left|\sum_{d \mid n} *_{\phi_{n / d}} x_{d}\right|^{2} \leqq \text { const. } \sum_{m=1}^{\infty}\left|x_{m}\right|^{2}
$$

If

$$
x_{1}=1, \quad x_{2}=x_{3}=\cdots=0 \text {, }
$$

this gives

$$
\sum_{n=1}^{\infty}\left|{ }^{*} \phi_{n / 1}+0+0+\cdots\right|^{2} \leqq \text { const. }\left(1^{2}+0+0+\cdots\right),
$$

which proves $(43)$.

\section{REFERENCES}

1. H. Bohr, Ueber die gleichmässige Konvergenz Dirichletscher Reihen, J. Reine Angew. Math. vol. 143 (1913) pp. 204-211.

2. - Fastperiodische Funktionen, Ergebnisse der Mathematik und ihrer Grenzgebiete, vol. 1, no. 5, 1932.

3. H. Burkhardt, Entwicklungen nach oscillierenden Functionen und Integration der Differentialgleichungen der mathematischen Physik, Jber. Deutschen Math. Verein. vol. 10 (1908) pp. 905-906. 66-74.

4. P. Hartman, On a class of arithmetical Fourier series, Amer. J. Math. vol. 60 (1938) pp.

5. E. Hellinger and O. Toeplitz, Grundlagen für eine Theorie der unendlichen Matrizen, Math. Ann. vol. 69 (1910) pp. 289-330.

6. A. Töpler, Grazer Mitteilungen, 1872, pp. 66-112 (the complete reference is not available here); a detailed abstract is given in [3].

7. O. Toeplitz, Zur Theorie der Dirichletschen Reihen, Amer. J. Math. vol. 60 (1938) pp. 880-888.

8. A. Wintner, Ueber die Spektra der Toeplitzschen D-Formen, Monatshefte für Mathematik und Physik vol. 48 (1939) pp. 147-152.

9. ——, Diophantine approximations and Hilbert's space, Amer. J. Math. vol. 66 (1944) pp. 564-578.

10. — On Töpler's wave analysis, Amer. J. Math. vol. 69 (1947) pp. 758-768.

The JohNS Hopkins University, BALTIMORE, MD. 